\title{
Tangence
}

\section{Montréal et Vancouver, métro-pôles d'un univers : L'hiver de Mira Christophe de Pierre Nepveu}

\section{Pierre Gobin}

Numéro 48, octobre 1995

Montréal et Vancouver : parcours urbains dans la littérature et le cinéma

URI : https://id.erudit.org/iderudit/025858ar

DOI : https://doi.org/10.7202/025858ar

Aller au sommaire du numéro

Éditeur(s)

Tangence

ISSN

0226-9554 (imprimé)

1710-0305 (numérique)

Découvrir la revue

Citer cet article

Gobin, P. (1995). Montréal et Vancouver, métro-pôles d'un univers : L'hiver de Mira Christophe de Pierre Nepveu. Tangence, (48), 10-23.

https://doi.org/10.7202/025858ar d'utilisation que vous pouvez consulter en ligne. 


\section{Montréal et Vancouver, métro-pôles d'un univers : L'hiver de Mira Christophe de Pierre Nepveu}

\section{Pierre Gobin}

L'biver de Mira Christophe, de Pierre Nepveu, publié par les éditions Boréal Express en 1986, n'est pas un "gros roman": à peine plus de deux cents pages. Son action apparente est "chargée de peu de matière , et ses personnages peu nombreux; le titre établit clairement temporalité et protagoniste. Pourtant ce texte lisible est aussi éminemment scriptible. En tout cas, il incite à la relecture: chacun de ses segments est apte à tourner et à changer l'éclairage de l'ensemble. Pour ne prendre que le titre, "l'biver" assume un éthos élusif (comme "the winter of our discontent" de Shakespeare, comme l'hiver de Mallarmé ou de Ducharme il est prison, mais aussi recueillement, ressourcement). Quant au nom de "Mira Christopbe", qui n'a rien de surprenant lorsqu'on se rend compte que l'héroïne est haitienne, ainsi que le Roi son homonyme, il évoque un regard et une prise en charge: un regard impératif, une charge messianique, la découverte d'horizons nouveaux, la traversée des eaux du désespoir. La relation ostensible d'amours brisées et d'illusions perdues que livre une première lecture du texte romanesque exige dès lors une attention nouvelle à des indices sur lesquels on aurait pu passer trop vite (le récit de l'avortement "dans" une tempête de neige à Montréal - qui renvoie à un autre hiver, celui de la tentative de noyade à Vancouver-Dollarton, et leurs variations fantasmées) qui incitent à penser à la nature du fardeau que porte Mira Christopbe, ainsi qu'à celle du regard qu'elle doit y diriger.

Si cela ne suffisait, l'auteur "annonce la couleur" dès le début de son entreprise. De même que, dans les romans de Calvino, nous devons projeter une partie de tarots et lire dans les cartes pour démultiplier des destini incrociati combinant aléatoire et programmation, dans le roman de Nepveu nous devons "rebattre" des bribes d'informations pour repenser la "croisée" de destins singuliers en "fenêtre " brisée dont les éclats réfractent des possibles pratiquement illimités, encore que l'attribution de tel ou tel chapitre à la visée de tel ou tel personnage nous renseigne sur le 
programme où nous devons entrer, et cela d'autant plus que les noms propres n'ont rien de gratuit. Voici l'incipit du livre (attribué à Etienne, personnage témoin, premier "professeur" christophorique, premier martyr lapidé, dont la fête suit symboliquement celle du Christ):

Le Livre d'Albert Mathieu (en mille morceaux jamais rassemblés, jamais écrits) dit que l'explosion est notre mère, big bang élémentaire dont nous sommes les éclats. Et puis après? Après, il y a l'éternité du temps, la mémoire qui flanche ou qui sévit, les bribes qui s'agglutinent, les formes qui gagnent. Albert Mathieu pense que c'est cela qui est le plus étonnant: qu'il y ait malgré tout des formes. Ça se brise, ça se refait autrement ${ }^{1}$.

Et l'épilogue du roman, attribué au même Étienne, commente la dernière lettre qu'il a reçue de Mira en ces termes:

Le Livre d'Albert Mathieu dirait que les désastres sont innombrables mais qu'il y a peu de ruines... (p. 218-19)

On le voit, le récit se construit, déconstruit, reconstruit, à travers une série de regards qui mirent et font miroiter l'histoire exemplaire d'un passage:

$\mathrm{Au}$ fond de soi (c'est toujours Etienne le "témoin lapidé "qui " épilogue v) les débris se relèvent, ou tourbillonnent, ou scintillent dans un fleuve qui ne s'arrête pas. (ibid)

Une fonction particulière est attribuable aux personnages, tant ceux dont l'aventure est relatée, Mira et Jean-René son amant, qui du reste la "réfléchissent " par une série de retours sur elle, que ceux qui la médiatisent dans le texte et bors du texte (ou du moins y interviennent en vertu d'intertextualité complexes) ${ }^{2}$. Etienne lui-même, Albert Mathieu, "personnage hy-

1 Toutes les citations renvoient aux pages du roman, édition de 1986. Les italiques renvoient aux réflexions du commentateur $P$. Gobin, mais parfois font partie du texte de $P$. Nepveu: dans ces derniers cas je le signalerai en note.

2 Il y aurait lieu de théoriser les "métissages" et les "manipulations géno-textuelles " qui font intervenir des productions symboliques d'ordres différents depuis l'ecphrasis du tableau par le texte jusqu'au festin d'Ésope d'Apollinaire, depuis le travail des Mots dans la peinture examiné par Butor jusqu'aux diverses formes d'Opéra, convergentes - le Gesamtkunstwerk de Wagner - ou divergentes dans des productions plus modernes. Pour ce qui est du roman de Nepveu, des exemples particulièrement intéressants sont offerts par les réflexions de Mira (qui a un album où elle croque l'image de ce qui l'entoure) sur l'œuvre d'Emily Carr (laquelle, on le sait, est aussi écrivaine - (et même poète au sens plein) - pp. 18-19), ainsi que l'épisode 
bride" de Montréalais vancouvérien, anglicisable en Al Matthew dont l'histoire en abyme occupe tout un chapitre (p. 123-136), ainsi que les confidents de Mira, sa nourrice demeurée en Haïti, son "nourrisson"/patient, le vieux millionnaire irlandais John Mac Duff, tous deux "insulaires", ne sont pas des comparses mais établissent le canevas de l'action et tissent le texte tout en le déconstruisant. En outre, derrière leur tapisserie de Pénélope, d'autres dessins se nouent. Pour revenir à la métaphore première que Nepveu privilégie, derrière leurs jeux de miroirs, d'autres visions se dessinent et/ou s'esquissent: celles de Malcolm Lowry ( $A u$ dessous du volcan est explicitement cité p. 29-30) et Gilbert Larocque (Les masques, placé sous le signe des Kindertotenlieder de Rückert/Mahler), tout comme des Livres célèbres dans l'absolu, en quelque sorte, (Le Livre de Mallarmé, L'influence d'un livre d'Aubert de Gaspé) "travaillent" le livre hypothétique d'Albert Mathieu, de même que les productions de ses patrons, petit Albert occultiste, Saint Albert le Grand, Docteur Magnifique auteur de sommes et, à l'horizon, le Livre de la Révélation.

Mais ces jeux intra/intertextuels par lesquels se font, défont, refont canevas et broderies, reflets et diffractions, voix et réverbérations ou échos, ne prennent leur sens qu'en s'exerçant sur les autres catégories aristotéliennes, et, au premier chef, le temps et le lieu ${ }^{3}$. Je me bornerai ici à l'examen de ce dernier, et concentrerai même mon attention sur les deux "pôles" de l'épisode central. Je ne considérerai donc pas les îles "perdues" dont Mira nourrit sa nostalgie, son Haïti natale, lieu de l'enfance près de Choupette et de l'a option pour la sainteté " au couvent des

où, bien que non musicienne, elle danse sur un disque de Debussy La fille aux cheveux de lin, inspiration paradoxale pour une jeune Noire, (p. 145146)), ou encore lorsqu'elle quitte le lieu de ses amours, la façon dont elle le "neutralise":

[Jean-René]... ne parvenait plus à présent à quitter ce lieu.... il s'était rendu compte, sans aucune surprise, qu'elle avait emporté Frauenleben und Liebe la musique qu'elle préférait mais il n'y avait en lui ni nostalgie ni attachement particulier pour l'endroit. (p. 197)

3 Aristote propose dix catégories (Kategoriai, section 4), qui font intervenir la substance, la quantité, la qualité, la relation (avant de passer au lieu, au temps, à la position, à la possession, et enfin à l'action et à la "passion"). C'est évidemment le lieu ( $5^{\mathrm{e}}$ catégorie) qui nous intéresse ici mais ce lieu est "flanqué " de la relation et du temps qui le "modulent "dans le roman de Nepveu. L'étude d'Émile Benveniste sur les schèmes de pensée aristotéliens ("catégories de langue et catégories de pensée") est particulièrement éclairante. 
religieuses françaises où elle a été éduquée (p. 43), ni l'Irlande mythique du vieux Mac Duff, non plus que les "étapes " immédiatement antérieure (New York) et postérieure (Oakland en Californie) du cheminement individuel de la protagoniste. Montréal et Vancouver, ces métro-pôles ont une tout autre importance puisqu'ils définissent les sites, l'unité dans l'opposition même, de l'un/ivers de la rencontre et de la séparation du couple formé par Mira et Jean-René, l'unique biver où leur histoire d'amour va connaître sa crise. La grande ville du Pacifique est le lieu même où culmine cette crise-anagnorèse (p. 16, Etienne évoque "le quartier de Kitsilano. Mira et Jean-René y ont vécu presque une année", et plus tôt il parlait des "quelques saisons en enfer " ${ }^{4}$ partagées par les deux amants dans un lieu pourtant "magique"). Mais le couple s'est rencontré à Montréal; Montréal sert de pierre de touche à leurs expériences de l'hiver vancouvérite; il est aussi le lieu du "regard", pour Étienne le narrateur qui "longe la rivière des Mille Isles" et qui se souvient de son propre voyage jusqu'au Pacifique (sur une "route onirique" pancanadienne qui retrouve les errances étatsuniennes de Jack Kerouac ou de Jacques Poulin), tout comme pour Albert Mathieu, le curieux "prophète"/transfuge de son pays québécois/étranger à sa cité d'adoption, qui "voit" Vancouver à travers Montréal et vice-versa.

\section{**}

Les deux grandes villes constituent pour Mira deux escales au nord du quarante-cinquième parallèle : entre New York, où elle a mené fiévreusement ses études d'infirmière, et Oakland où elle cherchera un dérivatif à ses "propres monstres" (p. 215) en travaillant aux urgences au sein d'une "tempête permanente", elle a cru pouvoir jeter l'ancre dans ces havres canadiens et y trouver la paix dans sa profession. Pour elle, Montréal ou plutôt l'Hôtel Dieu où elle exerçait, fut d'abord comme un refuge de "pudeur et

4 Toutefois, le souvenir rimbaldien ne doit nullement nous induire à penser que le couple était formé de "la vierge folle et l'époux infernal". Il est hétérosexuel même s'il est hétérogène et hétéroracial. Du reste ce n'est pas dans la sexualité que réside la folie de Mira (si folie il y a); et ce que Jean-René a "d'infernal " s'exerce en un lieu "clos" où Mira n'a pas accès, le centre de primatologie de Kitsilano. 
14

silence" (p. 20); à Vancouver, elle a "surtout connu des malades tranquilles" (p. 214) tels que le vieux Mac Duff.

Mais, à Montréal la rencontre avec Jean-René constitue une onde de choc au sein d'un espace quasi-immobile: lorsque le jeune homme est conduit d'urgence à l'Hôtel Dieu lors d'une crise d'appendicite aiguë - laquelle "frappe comme un coup du destin" (p, 20) - c'est elle "cette infirmière au visage noir qui veille [...] [son] insomnie post-opératoire.. À Vancouver, la rupture entre les amants est soulignée elle aussi par un remous "il valait mieux partir et devenir peut être une épave (terme significatif que je tiens à souligner) plutôt que de continuer ainsi" (p. 193). Les deux arrêts canadiens qui auraient pu constituer pour Mira des ports d'attache privilégiés sont en somme des lieux où elle est menacée "que sa quille éclate" sous les coups de la passion. Au reste, lorsqu'elle quitte Vancouver, il faut "[qu'elle] aille à la mer" comme eût dit Rimbaud. ("elle voyageait depuis un mois dans le nord, le long des fjords...". (p. 200)). Mais, paradoxalement, le roman ne nous montre pas l'activité portuaire en tant que telle, ni pour Vancouver ni pour Montréal : c'est en relation avec la "navigation" de la protagoniste que ces villes à la fois maritimes et fluviales sont présentées. L'eau, souvent évoquée, est d'ailleurs associée à des lieux écartés, calmes en apparence, et pourtant menaçants. Pour Montréal il s'agit non pas du vaste Saint-Laurent mais de la Rivière des Prairies, en marge de l'espace urbain, et ce cours d'eau, près duquel demeure Etienne, offre à ce dernier une métaphore pour l'ensemble du livre:

Je converge en m'éparpillant, je demeure par la seule erre d'aller, semblable à ma paisible rivière qui, cette fois comme chaque printemps, sort violemment de son lit et menace de transformer ma maison en arche de Noé.

Pour le témoin-relativement passif, qui renonce à son poste d'enseignant et ne cherche que mollement à intervenir dans son milieu ou auprès de ses amis - c'est la maison qui constitue le navire, et un navire-asile. Mira, active et volontariste, est ellemême le bateau. Souvent elle se jette dans le mouvement, la danse $^{5}$, la danse s'identifie avec des vagues, le mouvement proli-

5 Il serait intéressant de relever tous les épisodes où elle se meut ainsi. Ce qui me frappe c'est que c'est au bord de l'eau qu'elle le fait par prédilection. Nous avons déjà cité le ballet qu'elle improvise sur un disque de Debussy près de la Rivière des Prairies; à Montréal, lorsqu'elle "tombe en amour ", elle 
férant des saumons du Fraser (qui ne s'engouffrent dans le fleuve que pour y aimer et mourir, "possédés qu'ils sont par un mal qui ne pardonne pas. Mira a cru les entendre un jour [....], le frétillement de leurs milliers de queues bourdonnant comme un essaim d'abeilles au Sud de Vancouver" (p. 36)). Mais c'est dans une eau quasi-stagnante qu'elle est prête à se couler, à couler :

Dollarton! quelque chose s'est joué là bas, au bord de ce qui ressemble à un lac, en réalité l'extrémité du bras de mer qui s'enfonce dans les montagnes depuis Vancouver. C'est là que Malcolm Lowry a jadis arrêté son errance... (p. 28)

Le grand romancier de Sous le volcan a pensé que cette brûlure au milieu de lui-même n'était plus supportable et que seule pouvait l'en guérir:

son immersion définitive dans l'eau glacée (p. 28),

et il cherche à plonger sa persona romanesque :

non [dans] l'eau mais [dans] la légèreté [....] la promesse de légèreté.... Certitude de clarté, promesses de légèreté, de lumière, légère, lumière, lumière, lumière (p. 30$)^{6}$.

Mira, plus dynamique, moins introspective, se met à l'eau dans un mouvement analogue au lancement d'une embarcation.

Mira se tait puis soudain elle s'élance, elle court comme une petite fille que l'on n'a pas surveillée et qui dévale la pente vers

"court et virevolte"... "[son esprit] vagabond flotte sur l'étang [affreusement pollué du Parc Lafontainel, y plonge...." (p. 64); à Vancouver, elle parcourt plages et parcs longeant l'océan.

6 Ce passage (en italiques) est une citation directe. On notera cependant que la traduction "diffracte" le morphème anglais light en légère et lumière.

Il y a ici une "insoutenable légèreté" du jeu entre les textes, au niveau de la traduction, mais aussi du rapport entre les "statuts" des figures inscrites dans le roman de Nepveu:

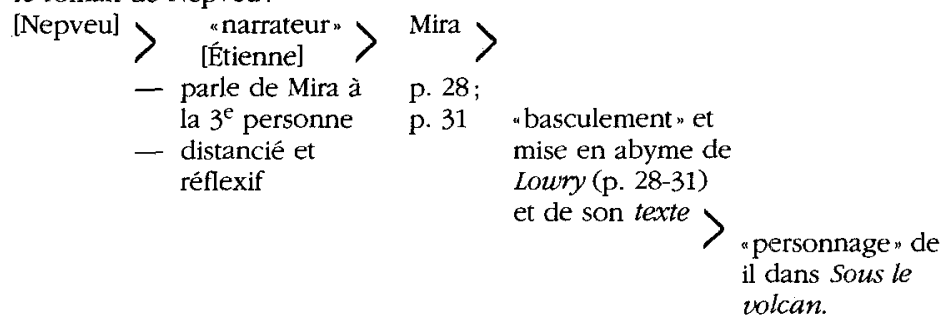

Cette admirable exploitation des ressources intertextuelles pour enrichir l'horizon narratologique mérite une étude particulière. 
la liberté. Le bruit de ses bottes sur le quai de bois. Son corps qu'elle laisse glisser dans l'eau froide un geste théâtral, irréel. Elle-même ne sait plus comment elle a pu le commettre... (p. 31)

Même si Jean-René réussit à "soulever "Mira hors de l'eau ("le fallait-il "s'interroge l'insaisissable instance éditoriale ici), dès ce moment le couple s'est défait, la communication est rompue:

Tu saisis le bras, tente (sic) de tirer Mira hors de l'eau, mais elle résiste, fait la morte, épouvantablement inerte. Mira je t'aime. Elle ne peut entendre?

Jean-René, on le remarque, est resté à quai ou à terre et c'est là qu'il tire la quasi-noyée. Son voyage de Montréal à Vancouver s'est fait par voie terrestre (et son retour aussi avec le camion de déménagement) ${ }^{8}$. Serait-ce que, voué à un support solide, il ne peut vraiment rejoindre celle qui a des affinités avec l'eau, les saumons, les embarcations? Est-il le lourd "chevalier" de cette Ondine, trop limité par son humanité pour percevoir les implications d'un environnement naturel? Ce n'est pas si simple. S'il ne se risque pas sur l'eau et n'est pas fasciné par l'eau, ce n'est pas qu'il soit un "terrien" borné, rivé au plancher des vaches. C'est que son exploration passe par une observation des franges de l'humain. Cette observation peut s'exercer aux confins de l'espèce, de ses contacts et de ses affinités avec de lointains "cousins" - à travers son métier de primatologue il est même devenu "une sorte de boulimique de l'observation" (p. 91), cherchant un "êtrange refuge" auprès des macaques lorsque l'espace se creuse entre Mira et lui - ou porter sur les humains se mouvant sur un

7 On peut alors lire Mira/Moïra, figure "noire" vouée au destin, échappant à la sphère humaine et aux prières. Quant à "l'instance éditoriale * qui s'adresse rétrospectivement à Jean-Marc ("comment es-tu parvenu ") mais qui se fait impersonnelle pour le questionnement du fatal ("le fallait-iln), elle peut être aussi bien l'amant lui-même, réfléchissant après coup sur l'événement, qu'Etienne, l'auteur, ou le destin qui interrogent encore le lecteur.

8 Pour le voyage Montréal-Vancouver Mira et Jean-René sont ensemble en auto. Mais je crois que cette objection n'exclut pas l'affinité de Mira avec l'élément liquide. Après tout Ondine fiancée au chevalier (chez La Motte Fouqué ou Giraudoux) s'est constitué des jambes, et danse sur la terre ferme. On remarquera que si Étienne évoque son propre voyage de Montréal à Vancouver aller et retour par la route, Albert Mathieu rêve d'emprunter la voie des airs ("plus glorieusement") pour être prophète en son pays (mais ce retour ne peut être triomphal, et devant Montréal "Albert se sent comme un ballon qui se dégonfle" (p. 129)). 
espace terrien. Si la jeune femme, au contact de la terre et de l'eau, contemple l'élément liquide et sa traduction dans le mouvement animal des saumons, son amant jette un regard à la RobbeGrillet ${ }^{9}$ sur les traces de ce mouvement sur le solide. Et Mira éprouve un malaise lorsqu'elle s'en aperçoit :

Dans une lettre, Mira parle d'une promenade à huit heures du matin sur une plage du parc [....] Jean-René se taisáit, et, un moment il s'est retourné pour regarder la longue courbe que la trace régulière de leurs pas avaient laissé derrière eux sur le sable dur. Il a souri et Mira dit qu'elle a détesté ce sourire... (p. 17)

\section{**}

La rencontre des amants et leur séparation sont donc modulées par des crisés existentielles: l'appendicite aiguë de Jean-René et la présence salvatrice de Mira; le glissement de la jeune femme dans l'eau du Pacifique et l'intervention de son amant pour l'en tirer; l'enfant que Mira a "failli avoir" (p. 55) et l'avortement (p. 112); l'accouchement de la guenon que surveille Jean-René

9 Pensons à Instantanés par exemple. Pensons aussi à la célèbre chanson de Prévert et Kosma (surtout à sa "chute " poignante):

Et la mer efface sur le sable

Les pas des amants désunis.

À l'instant où Jean-René sourit, le brouillard masque et les montagnes et la haute mer; il oblige à un regard d'anthropologue et non de navigateur; il permet au jeune primatologue d'assumer plus ou moins consciemment sur son amie une sorte de supériorité qui la blesse. Lorsque le brouillard se déchire et permet au regard d'embrasser l'Océan (p. 18) les amants s'embrassent doucement. Mais Mira a éprouvé une espèce de frisson devant l'espace qui s'ouvrait

Mira dit qu'il y avait quelque chose de colossal et de froid dans ce paysage.

Par ailleurs le mouvement migratoire des saumons vers le lieu de l'accouplement évoque la dynamique originaire de la conception (rencontre des gamètes des deux sexes dans le canal féminin, frétillement des spermatozoïdes), alors que les observations de Jean-Marc sur la naissance considèrent le terme de la gestation (là où les mâles n'ont plus de place, les macaques ne peuvent que menacer les femelles, comme le regard du a primatologue dans le couloir rouge. (p. 94) - encore une réminiscence d'Après la boue de Gilbert Laroque? - Jean-Marc lui-même est un témoin êtranger de l'avortement de Mira (p. 112), voyage "dur et solitaire, et sans surprise"). 
au cours de "la nuit des grands singes", et que domine "l'odeur unique et barbare de la naissance" (p. 93). La rencontre en un un/i/vers est aussi surdéterminée par la nature (éléments liquide et solide, saisons, rythmes du jour, rythmes de la vie). Pourtant ce sont les villes mêmes qui détiennent sans doute le secret de leur accord et de leur désunion. Encore une fois c'est Etienne qui suggère le lieu paradoxal de ce jeu entre deux lieux historiques et culturels

... l'écho de Montréal résonne autant que l'on veut dans le ciel de Vancouver et ces deux villes si peu sœurs et complices deviennent voisines... (p. 22)

Et plus loin, il passe comme physiquement de la perception kinétique d'une ville à une autre ( j'erre,... je traverse... je longe... [des lieux familiers de Montréal] [...] de la rive [de l'étang du parc Lafontaine] au rivage [du Pacifique] il n'y a qu'un pas [...] je flâne l'instant d'après sur la plage de la Baie des Anglais, je remonte la Strasse... puis je franchis le pont vers Kitsilanon). Même si son errance qui enchaîne les espaces urbains s'exerce au fil d'une "rêverie", elle est la clé d'un savoir. De plus, Étienne éloigné dans la durée (par la réminiscence) et dans l'espace (puisqu'il écrit au bord de la Rivière des Prairies) est pourtant présent, avec les amants. Non seulement $i l$ sait qu'il sait, mais il situe sa connaissance (détachée) au couur même d'une expérience avec les protagonistes dont toutes les données sont prises en charge. C'est son Montréal pourtant aliéné ("J'erre comme un fou dans les couloirs aseptisés de l'hôpital Notre-Dame,... je longe l'étang lisse comme un miroir...") - je souligne - qui lui permet de baigner dans un Vancouver lointain mais quasi-édénique. Sa conscience est alors globale comme il le laisse entendre:

Certain que je ne me suis pas trompé de lieu et de temps et que Mira et Jean-René sont bien ici, du côté de la Septième Avenue, dans une maison à pignon entourée d'arbustes, de rosiers et de cerisiers japonais. (p. 23)

Ce qui catalyse la "réaction" d'une ville sur l'autre que perçoit Étienne, c'est un dessin de Mira représentant sa demeure de Vancouver, mais qui semble prolonger le carnet de croquis qu'elle avait constitué à Montréal. Dans un cas comme dans l'autre, la jeune femme n'a accordé qu'une place insignifiante à ce qui pourrait vivre dans la ville ("comme toujours elle a bâclé les arbustes... aucun personnage aucun signe de vie»). En revanche, elle s'est efforcée de rendre la texture des matériaux. À 
Vancouver, "le bois frémit, se plisse, se teinte de rose et d'orangé"; à Montréal, "c'était toujours la brique, rouge, beige, ocre, qui sollicitait le plus son attention". Étienne suggère alors une explication :

comme si elle compensait ainsi la vie qu'elle ne pouvait reproduire ailleurs. (p. 19)

Serait-ce à dire que la jeune femme se conçoit comme stérile? Pourtant il semble bien que ce soit en vertu d'une décision qu'elle n'a pas d'enfant ("la naissance n'appartient pas au plan. Un accident incompréhensible" se rappelle Jean-Marc avant d'évoquer le "dur et solitaire" voyage de Mira dans l'avortement, et si l'interruption de grossesse a été spontanée, "de toute façon c'était ce que tous deux avaient souhaité sans oser le dire" (p. 113) $)^{10}$. Et si elle se borne à une notation conventionnelle des végétaux dans ses dessins, elle soigne avec amour des géraniums, qu'elle confie à son amant lorsqu'elle le quitte ("Devant la fenêtre les trois pots de géraniums. Il remarqua que Mira les avait arrosés

10 On pourrait être tenté de construire un carré sémiotique associant les quatre personnages clés (protagonistes et confidents) à une relation particulière envers la vie et la mort.

protagonistes

impossible perpétuation de la vie

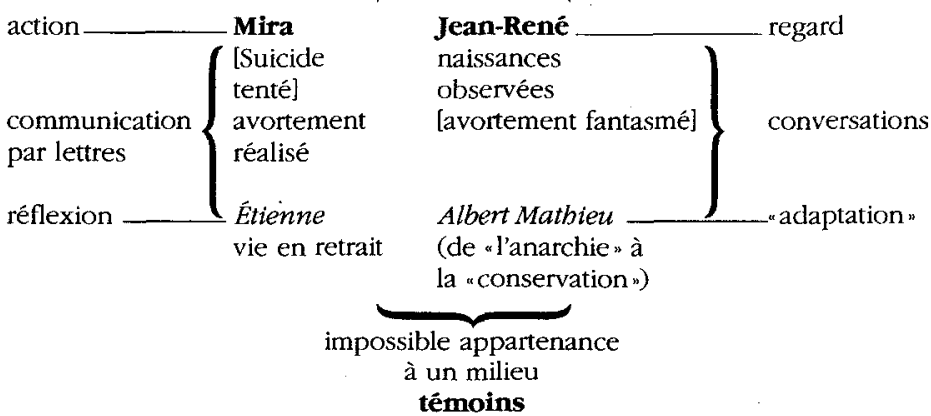

Cette relation rejoindrait du reste une perception spécifique des éléments qui, curieusement, serait lisible en termes bachelardiens: on y verrait associés Mira et l'eau, avec une scène traumatisante et "ophélique ", Jean-René et la terre, avec un projet - "rêveries de la volonté " - à l'opposé des "rêveries du repos "d'Étienne, Albert et les songes "aériens "; il manquerait toutefois le $f e u$, à moins que le travail sourd de l'œuvre de Lowry n'évoque celui qui couve Sous le volcan; du reste le projet de Bachelard articulait les quatre éléments en cing. 
abondamment avant son départ". p. 194) Il y a donc, dans les réaction de la jeune femme à la ville une réaction presque analogue à celle qu'elle a devant la vie. L'énigme de son attitude envers Vancouver peut être abordée par là. Les maîtres-mots de son expérience, qu'elle "trahit" par l'insertion dans ses lettres de "majuscules nerveuses qui éclatent au milieu d'une page toute en douceur. ATTENTION, AMOUR, ART, DESTRUCTION, et surtout VANCOUVER" (pp. 12-13) semblent au départ des paradoxes. Pourquoi être "hantée" par "cette ville qu'elle a quittée il y a plus d'un an"? Pourquoi être fascinée par l'amour après une rupture et un déchirement qui devrait du reste se cicatriser? Et peut-on parler de destruction à propos de Jean-René? Étienne s'interroge:

Lui, un destructeur? le raisonneur lunatique, le scientifique aux yeux tendres, sourcils inquiets, bouche cousue? (p.13)

Un élément de réponse est sans doute donné par la protagoniste au chevet de MacDuff mourant. C'est à ce vieillard à la fois aussi étranger et aussi proche qu'on peut l'être, riche, blanc, vieux, mais aussi insulaire et attentif aux grands passages existentiels, beau-parleur ("I kissed Blarney's stone" dit-il curieusement, p. 189) et réduit au silence, si avide de vivre et si proche de l'état de fantôme qu'elle se confie et elle se refuse:

Que penserais-tu de moi vieux John, si tu m'écoutais vraiment? (p. 45-46)

ou encore:

Ne m'écoute pas John MacDuff. Tu ne me payes pas pour que je te raconte des histoires, surtout pas de celles qui se perdent dans l'espace inhumain du dedans. (p. 62-63) ${ }^{11}$

Une autre "distribution", qui permettrait d'éclairer les rapports, le rapport aux villes (aux deux villes): Mira en dessine les micro-éléments, en privilégiant activement les textures et les matériaux; Jean-René en observe les grandes masses et les activités (le cerf-volant sur la plage - p. 151-153; les skieurs - p. 180-181 à Whistler); Albert en conserve les artefacts après avoir été tenté d'en agiter les masses; Étienne réflécbit sur leurs interactions qui fournissent un arrière-plan qui n'est pas "pratico-inerte" comme pour Sartre, mais qui intervient dans un développement.

11 C'est précisément ce texte que Nepveu (et/ou son éditeur?) a choisi pour la quatrième page de couverture, et qui semble associer les cinq maître-mots, "arcanes majeurs" de ce jeu romanesque de destins croisés, susceptibles de redistributions - il faut les battre et les couper - et de réinterprétations nouvelles impliquant aussi leurs corrélats et leurs antonymes, leurs contraires 
Mais cette coquetterie tragique, ou ce mouvement ironique qui vise à la rencontre de l'Autre et du Même dans un un/ivers commun transpose l'expérience de Mira et de Jean-René (femme et homme, danseuse et observateur, Noire et Blanc, insulaire et continental, ondine et terrien), parfois au sein d'un seul individu (Albert/Al, le vancouvérien montréalais qui veut incarner ses deux "patries" alors que les partenaires sentent plus ou moins clairement que la conjonction parfaite, la communication pleine sont impossibles). Et c'est précisément sur cette attraction-fascination, (ATTENTION, AMOUR, ART) que se construit la relation entre les deux villes (DESTRUCTION - et surtout VANCOUVER, que je souligne, puisque le texte s'adresse à des Montréalais, le destinataire immédiat des lettres de Mira, Étienne, et nous, lecteurs francophones du roman de Nepveu).

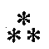

Dès lors les "Images" (p. 97-121) auxquelles s'accroche JeanRené, qu'il s'agisse de souvenirs (instantanés montréalais où figure Mira), ou de perceptions fugitives de Vancouver (où le jeune homme circule seul), de vignettes-cartes-postales

Devant lui, Gastown: Gassy Jack, tavernier de la belle époque, cherche des yeux la mer du haut de son socle (p. 121)

de séquences cinématographiques avec mouvement et son

(1). Mira debout, les poings sur les hanches, sur un balcon en plein été, elle s'indigne, vocifère à propos d'une enquête sur la discrimination raciale dans l'industrie du taxi à Montréal... (p. 104)

voire de clichés surimposés

(3). Hivers (au pluriel) qui déferlent, transportent Montréal en pays arctique. Poudrerie et lumière de glace.... (id. ibid)

ne peuvent constituer qu'un ensemble labile. On ne réussira pas à en constituer un album, à les monter en séquences filmiques classiques, les inscrire dans une ouvre d'ART stable, un AMOUR

et leurs contradictoires (par exemple destruction/déconstruction/reconstruction). Et quel peut être le terme complémentaire de Vancouver, sinon Montréal? 
stable, quelle que soit l'ATTENTION que l'on y apporte, quelle que soit leur puissance évocatrice. L'exemple le plus révélateur de cette distance ironique nous est offert par la scène où les amants en apparence totalement épris, couple idéal ("Tu es beau, Jean-René, dis-moi que je suis belle. Et que la beauté engendre la bonté..." (p. 151)) voient sur la plage le long de la Baie des Anglais une scène en apparence admirable:

Plus loin un Chinois ou un Japonais faisait monter et descendre un cerf-volant avec un art très appliqué et une parfaite concentration.... (p. 152)

Cette vision à la fois exotique et si typique de Vancouver, où le contrôle d'un artefact épouse et mime la nature, semble arracher les existants aux contingences:

Il devenait à ce moment précis le maittre du monde, le maître des vents, des mouvements et des chutes, réussissant non pas à faire voler un objet [....] mais quelque chose de bien plus difficile, une suspension entre le haut et le bas, un frôlement jusqu'à rass de terre et pourtant encore aérien....(ibid.)

Pourtant le cerf-volant est minable ("un vulgaire losange de plastique transparent..."), son maître si visible est ignoré de tous ("sauf un vieillard [....] qui l'aperçut et nous jeta un regard ironique"), et lui-même est comme abstrait de Vancouver, livré "à un fou-rire", ignorant tous les êtres qui l'entourent ("Jamais je pense il ne nous a vus" (p. 153)).

Si la rencontre avec l'art est précaire et comme dérisoire ${ }^{12}$, quelle chance peut avoir l'amour? quelle chance peut avoir la communication entre deux villes?

Et pourtant... Pourtant, le paquet de lames de tarots que constitue le Livre de Nepveu vous offre la chance de jouer sur des solitudes démultipliées, dont Mira et son amant, mais aussi Vancouver et Montréal sont les partenaires et les enjeux. Nous avons ainsi le roman le plus révélateur de l'identité clivée qui se

12 Ici toute la problématique du "saltimbanque", mise en évidence pour ce qui est de lart moderne par Starobinski se trouve réintroduite en termes postmodernes. Le "saut" d'une rive à l'autre du Canada, d'une rive à l'autre de l'éventail idéologique - agitation anarchiste/exploitation conservatrice d'une rive à l'autre de nos horizons culturels - québécois et canadien qu'effectue Albert Mathieu en est le témoignage. La façon de repenser Sous le exlcan offriratit une autre perspective pour l'explorer. 
cherche "d'une mare à l'autre" (Ferron dixit) depuis celui de Hugh MacLennan il y a une génération, où la visée montréalaise et la vision qu'est Vancouver se combinent et recombinent pour questionner le pouvoir des "capitales" (que les deux villes ne sont pas) et des "centres". 